\title{
Numerical Modelling of Sonicated, Continuous Transesterification and Evaluation of Reaction Kinetics for Optimizing Biodiesel Reactor Design
}

\author{
Mohammed Noorul Hussain, Tala el Samad, Mohammed Daqaq, Isam Janajreh * \\ Masdar Institute of Science and Technology, Abu Dhabi, UAE, 54224
}

\begin{abstract}
Biodiesel is an alternative and sustainable fuel that can reduce the dependence on fossil diesel. This commodity has not only been promoted in the developed world but also in Indonesia, Brazil and several developing counties. In a general procedure it is a product of a transesterification reaction of vegetable oils or waste cooking oils with an alcohol, in the presence of an acidic or basic catalyst. It is a slow reaction which is conventionally carried out in a mechanically stirred batch process. An advanced method to achieve high yield quality in less time is sonication of the reaction in an integrated continuous process. Sonication causes micro-cavitation in the reactant mixture. The cavitation bubbles can have an internal pressure and temperature as high as 1000 atm and $5000 \mathrm{~K}$, respectively. Violent collapse of these bubbles causes tremendous increase in mass transfer, thereby enhancing the reaction rates [1]. To optimize the design of the reactor, high fidelity modeling assisted design is pursued. This enables the effective integration of the reactant transport and the sonication effect in a coupled acoustic, reactive multiple-specie flow. In this work, a cylindrical reactor is considered in which reactant mixture will be circulated and sonicated by a sonotrode type ultrasound equipment. To simulate the sonication effect the linear, time independent wave equation is solved for the fluid domain, which provides us with the acoustic pressure variation in the fluid. To account for the attenuation of the wave due to cavitation bubbles the modified wave number is used. To account for the chemical reactions, laminar reacting flow is assumed for the reactant mixture for which the Navier-Stokes equations and transport equation for dilute species is solved for the fluid. A logical reaction rate coupling model, which is dependent on the acoustic pressure and flow velocity, is used to evaluate the kinetics of the reaction, which are to be used as a judging factor for the reactor design [2].
\end{abstract}

Keywords: Transesterification, ultrasound, biodiesel, process intensification, numerical modelling.

\section{Introduction}

Transesterification reaction is a mass transfer limited reaction, which is neither strongly endothermic nor exothermic. Theoretically and based on the process stoichiometry, 3 moles of methanol are required to convert 1 mole of TG to 3 moles of Fatty Acid Methyl Ester (FAME-biodiesel) and one byproduct mole of glycerol [3]. The reaction takes place in the presence of a catalyst that can either be homogeneous catalyst like $\mathrm{NaOH}$, $\mathrm{KOH}, \mathrm{H}_{2} \mathrm{SO}_{4}$ or heterogeneous catalyst like $\mathrm{CaO}$ as shown in Equation (1).

${ }^{*}$ Corresponding author. Tel.: +971 28109130

Fax: +9876543210; E-mail: Author.ijanajreh@masdar.ac.ae

(C) 2016 International Association for Sharing Knowledge and Sustainability

DOI: $10.5383 /$ ijtee.11.01.012

\begin{abstract}
Ways of making the process faster and more productive must be found to make biodiesel lucrative in the market. Chemical process intensification has been receiving much attention and can be applied to transesterification. In one of the multiple ways to achieve this is the option of pushing the reaction parameters such as temperature and pressure to reach supercritical conditions. However the energy consumption in this process is very high due to the elevated state conditions. Sonication is another pathway for speeding and intensifying the reaction. Sonication is simply the use of ultrasound to create pressure
\end{abstract}


variations in the fluid domain which causes growth of microbubbles in the bulk of the fluid. These bubbles have very high internal temperature and pressure ( $\approx 1000 \mathrm{~atm}, 5000 \mathrm{~K})$. At relatively high ultrasonic intensities these bubbles grow rapidly and collapse violently, generating energy and mechanical effect which in turn increases the overall mass transfer rate [1]. Sonication generates millions of such bubbles in the fluid, hence the combined action of the micro-level activities enhances the reaction in the macro fluid domain. This is a much simpler and energy efficient process.

Triglyceride +3 Alcohol $\frac{K 1}{K 2} 3$ FAME + Glycerol

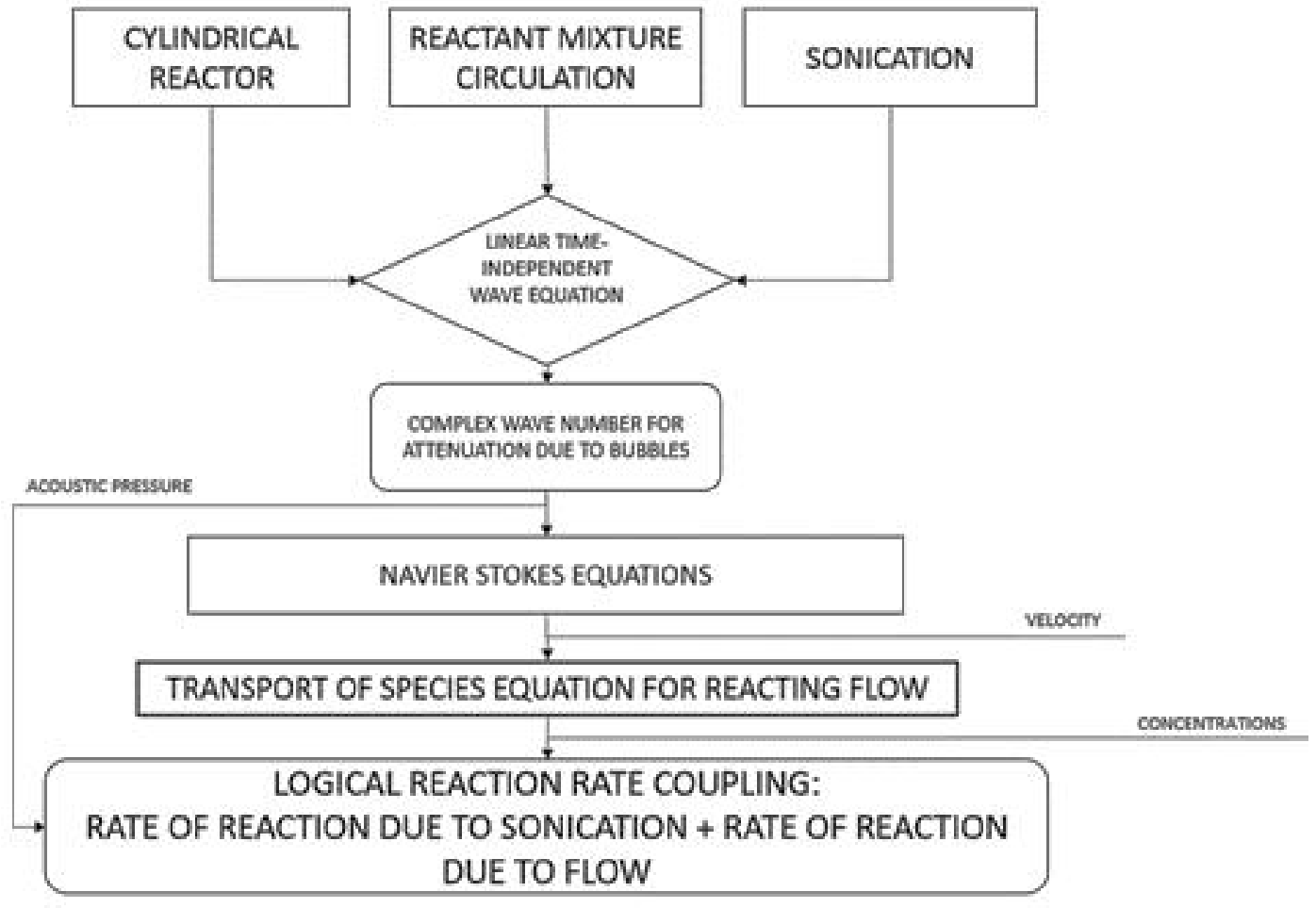

Figure 1. Schematic of the methodology

Compared to the conventional stirred method sonication has proven to be much more productive. For example Stavarache et al. [4] reported higher yields in shorter time using ultrasonic transesterification under homogeneous catalysts of $\mathrm{NaOH}$ and $\mathrm{KOH}$ and for the same molar ratio and catalyst amount compared to conventional stirring method. Manickam et al. [5] reported that mechanical agitation method requires triple the time to achieve $78 \%$ yield as compared to ultrasonic transesterification which gives $93 \%$ yield with $1 \% \mathrm{KOH}$ and $3: 1$ methanol to oil molar ratio.

A compact sono-chemical reactor which can give good yields of biodiesel in shorter time is a necessity. Such a reactor finds applications in commercial biodiesel production and laboratories for research on biofuels. Design and development of small scale sono-chemical reactors can help in designing large scale ones. Another idea behind designing such a reactor is to make the reactor viable for domestic utilization, such that the waste cooking oil generated from each home or community can be domestically converted to biodiesel and then be used by that home or community. Hence the reactor needs to be highly efficient, and inexpensive. The second aspect after choosing the method of production (sonication) that is crucial for the reactor design is to setup the reactor system control as far as processing the fluid in controlled mass batches or in continuous flow reactor. Designing the reactor for continuous production has several advantages in minimizing manual intervention and batch to batch variability and increase in production volumes.

In designing sono-chemical reactors the key feature is the the reactor geometry. Geometry should be such that it allows the wave to propagate freely without much damping. A limited works on numerical simulation and modelling have been reported on reactive flow coupled with sonication. This work is based on COMSOL Multiphysics for acoustic simulation [2][6][7][8]. Numerical simulation has been used to evaluate reaction kinetics of transesterification earlier [9]. A work by the same author which used Ansys FLUENT for reacting flow analysis of a sono-chemical reactor, made a comparison between sonicated and circulated flow reaction kinetics and showed that indeed sonication resulted in better yields. However acoustic simulation was not done in that work. [10].

In this study heuristic numerical simulation is done to evaluate the design in terms of acoustic propagation, reactant flow and chemical kinetics. COMSOL Multiphysics, which is a finite element based package is used in this study. The results from the individual studies are used logically to evaluate the reaction kinetics using the coupling model developed by Jeroen et al [4]. Later a parametric study based on temperature and molar ratio is carried out to understand the FAME formation trends in the reactor design. 


\section{Methodology}

The method followed to design the reactor is schematically shown in Fig.1. In this work initially two different geometries, each cylindrical in shape, were investigated for the best acoustic wave dispersion, so as to broadly evaluate the best reactor configuration. After selecting the reactor configuration, further analysis was done with a 2-D approximation of the design. Primarily, the wave equation was solved to find the pressure variation in the reactor, the complex wave number was applied next to estimate the wave attenuation in the reactor. For the reactant flow Navier-Stokes equations were solved which provided the flow velocity that used in the species transport equation to determine the resultant concentration. Using the results from the above steps reaction kinetics were evaluated for both sonication and circulation. These were then coupled logically, using inference from earlier works [4]. The core modules from the schematic are explained in detail.

\subsection{Linear time-independent wave equation}

The general wave equation which gives the pressure distribution in the medium is given in Eq. 2.

$$
\nabla^{2} \mathrm{P} \frac{1}{\rho}-\frac{1}{\rho \cdot \mathrm{c}^{2}} \frac{\partial^{2} \mathrm{P}}{\partial \mathrm{t}^{2}}=0
$$

Where $\mathrm{P}$ is the acoustic pressure and $\rho$ and $c$ are the medium density and the sound velocity, respectively. In order to simplify the problem, the non-linearity which is associated with the ultrasound waves is neglected. The density and speed of sound are assumed to be constant throughout the medium. The pressure is considered as time harmonic function $P(x, t)=P(x) e^{i \omega t}$. These assumptions simplify the equation to $\mathrm{Eq} 3$.

$$
\nabla^{2} \mathrm{P} \frac{1}{\rho}-\frac{\omega^{2}}{\rho \cdot c^{2}} \mathrm{P}=0
$$

The above equation was used for initial pressure distribution analysis to identify a relatively good design in terms of sonic energy utilization. For the wave attenuation the general Helmholtz equation is used as in Eq (4).

$$
\nabla^{2} \mathrm{P}+\mathrm{k}_{\mathrm{c}}^{2} \cdot \mathrm{P}=0
$$

Where $\mathrm{k}_{\mathrm{c}}$ is the complex wave number which accounts for the attenuation due to the cavitation bubbles.

\subsection{Complex wave number}

Cavitation bubbles caused by the compression and rarefaction of the liquid due to the ultrasound pressure are good for the mass transfer in that region but they also cause loss of ultrasound energy. This is called the attenuation of the wave [6]. This phenomenon is highly non-linear and attempts were made to model their growth and effect [11]. But the complexity of the modelling had to be reduced to make the designing process simpler and reliable. Commander and Prosperetti [12] worked on the linearization of the model developed by Caflisch et al [11]. They developed a linear approximation and converged to Eq. 5. The key component of the equation being $k_{c}$ which is the complex wave number. The assumption here is that the bubble radius is constant [6], and the $\mathrm{k}_{\mathrm{c}}$ given as:

$$
k_{c}^{2}=\frac{\omega^{2}}{c^{2}}\left(1+\left(4 \frac{\pi c^{2} n_{b} R}{\omega_{o}^{2}-\omega^{2}+2 i b \omega}\right)\right.
$$

Where $\mathrm{n}_{\mathrm{b}}$ is the number of bubbles, $R$ is the bubble radius and $b$ takes the damping effect parameter. $\omega_{0}$ is the resonance frequency and is defined as:

$$
\begin{aligned}
& \omega_{o}=\left(\frac{P_{0}}{\rho R^{2}}\right)\left(\operatorname{Re}(\emptyset)-\frac{2 \sigma}{R P_{0}}\right) \\
& P_{o}=P_{\text {liq }}+\frac{2 \sigma}{R} \quad \text { (Which is the undisturbed pressure) }
\end{aligned}
$$

Where $\sigma$ is the surface tension and $\varnothing$ is a complex dimensionless parameter and is given as:

$\varnothing=\frac{3 \gamma}{1-3(\gamma-1) i \times\left[\sqrt{\frac{i}{x}} \operatorname{coth}\left(\sqrt{\frac{i}{x}}\right)-1\right]}$

Where $\gamma$ is the specific heat ratio and $\mathrm{X}$ takes the value as:

$$
X=\frac{\mathrm{D}}{\rho \mathrm{R}^{2}} \quad \text { (Where } \mathrm{D} \text { is the thermal diffusivity) }
$$

The damping coefficient is described by:

$$
\mathrm{b}=\frac{2 \mu}{\rho \mathrm{R}^{2}}+\frac{\mathrm{p}_{\mathrm{o}}}{2 \rho \omega \mathrm{R}^{2}} \operatorname{Imag}(\varnothing)+\frac{\omega^{2} \mathrm{R}}{2 \mathrm{c}}
$$

And the cavitation bubble volume is evaluated as:

$$
\begin{aligned}
& \beta=\frac{4 \pi n_{b} R^{3}}{3} \\
& \beta=2 \times 10^{-9} P \quad \text { for } p_{\text {blake }}<P<1 \times 10^{8} P a .
\end{aligned}
$$

The Pblake is the blake threshold. This is the pressure above which the cavitation bubble will implode. This is further used in coupling the reaction rates, and $\beta$ which is maintained at 0.02 [2].

\subsection{Navier stokes for flow simulation}

In order to make the process continuous reactant, the mixture is made to flow in the reactor from inlet at the top to the outlet at the bottom. Vegetable oil and methanol are two immiscible fluids, but in a premixed state the solution can be assumed to have a uniform mixing of these two fluids in the reactor. Therefore the first assumption made here is that the mixture is homogeneous. Secondly, ultrasonic cavitation causes undulations in the flow profile due to shockwaves, pressure variations etc. However this model is a linear approximation hence does not integrate such effects. Considering these assumptions the laminar, 2D, incompressible, viscous Navier Stokes equations as given in Eq.13 is used to simulate the flow of reactants inside the reactor.

$$
\rho(\boldsymbol{u} \nabla \boldsymbol{u})=-\nabla P_{\text {flow }}+\mu \nabla^{2} \boldsymbol{u}+\rho \boldsymbol{g}
$$

Where $\boldsymbol{u}$ signifies the velocity component vector and $\mu, \boldsymbol{g}$ are the molecular viscosity and gravitational acceleration, respectively. The inlet velocity was kept low enough to avoid interference with the sound wave [4]. The velocity profile obtained from the resultant is used in the species transport equation. 


\subsection{Species transport}

Interaction between reactants occurring due to the flow of reactant mixture in the reactor promotes increase in transesterification. The reactor must be designed in a way that the flow is highly efficient in promulgating the reaction, such that high yields of biodiesel are attained even in absence of sonication. To analyze this aspect of the reactor, species transport equation is used to predict the resulting biodiesel concentrations within the reactor as shown in Eq.14.

$\boldsymbol{\nabla}(-D \boldsymbol{\nabla} c)+\boldsymbol{u} \boldsymbol{\nabla} c=R_{\text {rate }}$

Four species, vegetable oil, methanol, FAME (biodiesel) and glycerol are transported through the reactor. The reaction is defined by the Arrhenius kinetics model where the rate constant is defined as in Eq.15 and only the forward reaction is evaluated and is written as:

$k_{\text {flow }}=A \cdot e^{\frac{-E}{R_{u} \cdot T_{l i q}}}$

Where $\mathrm{E}$ is the activation energy and $R_{u}$ here is the Universal gas constant and $\mathrm{T}_{\text {liq }}$ is the fluid temperature. The activation energies $E$ were approximated from the work of Noureddini et al [13].

\subsection{Reaction rate coupling}

The most defining part in evaluating the design of the reactor is the total reaction rate of biodiesel formation. This is used as the judging factor for the reactor design. The total reaction rate is the logical sum of the reaction rate due to sonication and reaction rate due to reacting flow. The kinetic model developed by Jeroen et al [4] is used to quantify and sum the individual reaction rates. However the nonlinear effects of ultrasound such as the sonoluminescence, shock waves and formation of radicals is neglected in their model. Therefore, only thermal effect of sonication is incorporated in this model. The temperature inside the cavitation bubble is modelled as a function of the acoustic pressure assuming the vapor inside the bubble to be a molar mixture of oil and methanol vapors. The Arrhenius model is then applied to find the rate constant of sonication as shown in Eq.16 As the physical phenomena suggests the reaction rate due to sonication comes in to effect only with the implosion of cavitation bubble, the rate of reaction has to be a function of the cavitation bubble volume. But since the bubble implodes only when the acoustic pressure greater than the Blake threshold pressure, the sonication reaction rate equation should be such that it comes into account only when $\mathrm{P}>\mathrm{P}_{\text {blake. Eq. }} 18$ gives the total reaction rate evaluation equation.

$$
\begin{aligned}
& k_{\text {son }}=A \cdot e^{\frac{-E}{R_{u} \cdot T_{\text {bubble }}}} \\
& T_{\text {bubble }}=\frac{T_{\text {liq } P(\gamma-1)}}{P_{\text {vapor }}} \\
& -R_{\text {total }}=\left[\left(P>P_{\text {blake }}\right) \beta k_{\text {son }}+(1-\beta) k_{\text {flow }}\right] * \text { concentrations }
\end{aligned}
$$

The rate coupling model works on the physical fact that all the bubbles generated implode only at a certain pressure threshold (Pblake), hence they only implode in the areas where the acoustic pressure is higher than the threshold. Therefore the reaction rates $\mathrm{R}_{\text {total }}$ are high only in these areas.

\subsection{Boundary conditions}

For acoustic simulation a dirichlet boundary condition $\mathrm{P}=\mathrm{P}_{\mathrm{w}}$ was assigned at the end of the sonotrode implying the source of ultrasound energy dissipation, where $\mathrm{P}_{\mathrm{w}}$ is a function of the power $\mathrm{P}_{\mathrm{d}}$ as given in Eq.19

$$
P_{w}=\sqrt{\frac{2 \rho C \mathrm{P}_{d}}{A}}
$$

For all other walls a sound hard boundary condition $\mathrm{P}=0$ was applied.

For the flow simulation the velocity boundary condition was applied at the inlet, and dirichlet $\mathrm{P}_{\text {liq }}=0$ was applied at the outlet. All walls were subjected to no slip, $\mathrm{u}=0$ boundary condition. For species transport the inflow was given as molar concentrations.

\section{Problem Setup}

For the initial analysis three reactor geometries were considered as shown in Fig. 2 a, b. For the detailed analysis the 2D geometry shown in Fig. 2c was adopted. Geometry-1 followed a concentric cylinder shaped design, with the inner cylinder having a slight offset from the central axis to provide inclusion of the sonotrode (seen as a small cylinder in the geometry). The flow was supposed to occupy the annular region within the cylinders, with a tangential inlet at the top and outlet at the bottom. Geometry-2 was a single cylinder design which is analogous to placing a sonotrode in the center of a beaker, but with tangential inlet and outlet. Geometry-3 is similar to geometry-2 but with additional incorporation of a static mixer, and the inlet is not tangential. This design was adopted as an optimization in lieu of certain limitations of the ultrasound and the plain cylindrical geometry. A sonotrode type of ultrasound equipment generally can sonicate flows with very low flow rate, and a plain cylindrical design does not effectively help in mixing of reactants. Therefore a static mixture section is added to utilize the whole length of reactor in reaction propagation effectively. Having such a division causes some sonic concentration in the upper half of the reactor body, rendering it to be a pseudo ultrasonic flow cell section. In each case the height of the reactor was $25 \mathrm{~cm}$ and diameter was $6 \mathrm{~cm}$. The sonotrode diameter was $2 \mathrm{~cm}$. In geometry-1 the inner cylinder was $3 \mathrm{~cm}$ in diameter.

\section{Results and Discussion}

\subsection{Design selection by acoustic simulation}

The simulations were done for a frequency of 40,000 Hz. Analyzing the results shows us that the pressure profiles have a big difference. The peaks of pressure are obtained in the bottom part of the reactor geometry- 2 in a position opposite to the sonotrode. The maximum acoustic pressure was $4.85 \mathrm{e} 5 \mathrm{~Pa}$. The important observation to be made is here is that there is much energy being wasted due to the hollow section owing to the concentric cylinder design. This non-utilization of energy is not a good sign for a sono-chemical reactor. On the other hand for geometry-2 we see that though the maximum acoustic pressure is $1.53 \mathrm{e} 5 \mathrm{~Pa}$, the ultrasound effect is being distributed uniformly over the complete reactor domain. The reason for the peak in geometry- 1 being higher than the peak in geometry- 2 could be the reflection of the acoustic wave within the annular chamber of the reactor. However having annular chamber reduces the volume of the fluid being processed and also such a design 
reduces the time of interaction of the fluid and the ultrasound source. Therefore design geometry-2 was adopted for further optimization. Design geometry-3 is an optimized form of geometry-2. The results of acoustic simulation of geometries-1, 2 are shown in Fig. 3.

\subsection{Attenuation due to cavitation bubbles}

For the frequency $40,000 \mathrm{~Hz}$ the Helmholtz equation was solved with a normal wave number and the complex wave number. The attenuation of the wave is much evident as seen in Fig. 4. The peak pressure for both attenuated and un-attenuated conditions is common due to the common frequency. However the damping of the wave is completely different. The peaks in case of cavitation are limited to a small region just around the ultrasound source. The oscillation of pressure has highly diminished and can only be seen to a very small extent in Fig 4(a). This clearly demonstrates that the attenuation due to bubbles is very high. This has its own pros and cons. Having high number of bubbles means having more implosions thereby high reaction rate, however the attenuation of the wave limits the development of having bigger reactors. Increasing reactor size may mean having larger or multiple ultrasound sources, which is not good in the energy consumption point of view.

\subsection{Reaction evaluation and parametric study}

The inlet velocity was considered to be $0.0044 \mathrm{~m} / \mathrm{s}$ to have approximate residence time as $1 \mathrm{~min}$. The reaction rates for flow were calculated for 3 different temperature conditions and molar ratios. The $\mathrm{k}_{\text {flow }}$ and reaction rate showed direct dependence on the molar ratio and temperature. This analysis helped to determine that having a static mixer incorporated in the design is a good idea since FAME was seen to be forming between the mixer blades. Fig 5 gives the $\ln \mathrm{k}_{\text {flow }}$ vs $\left(1 / \mathrm{T}_{\text {liq }}\right)$ plot and Fig 6 . shows the FAME profile in the reactor at different molar ratios and Fig. 7 for different temperatures.

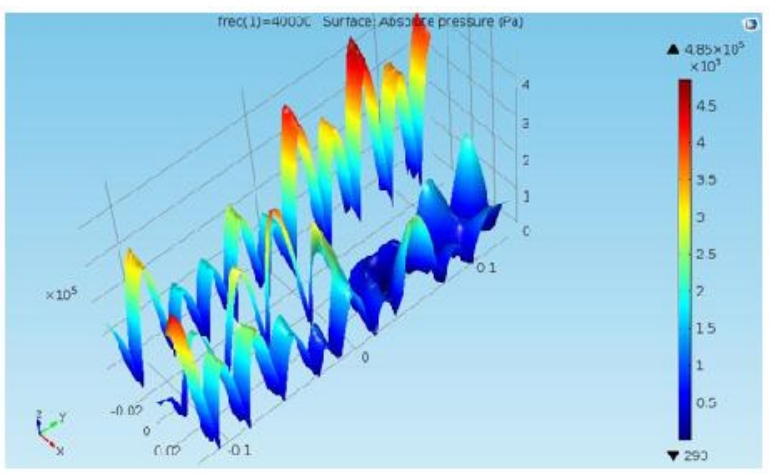

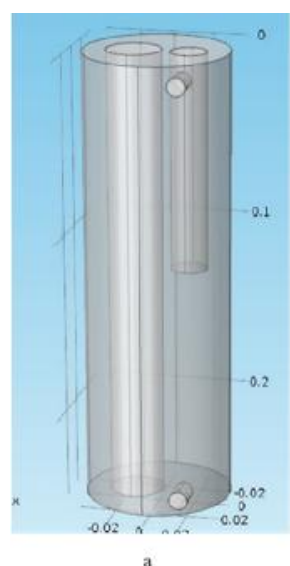
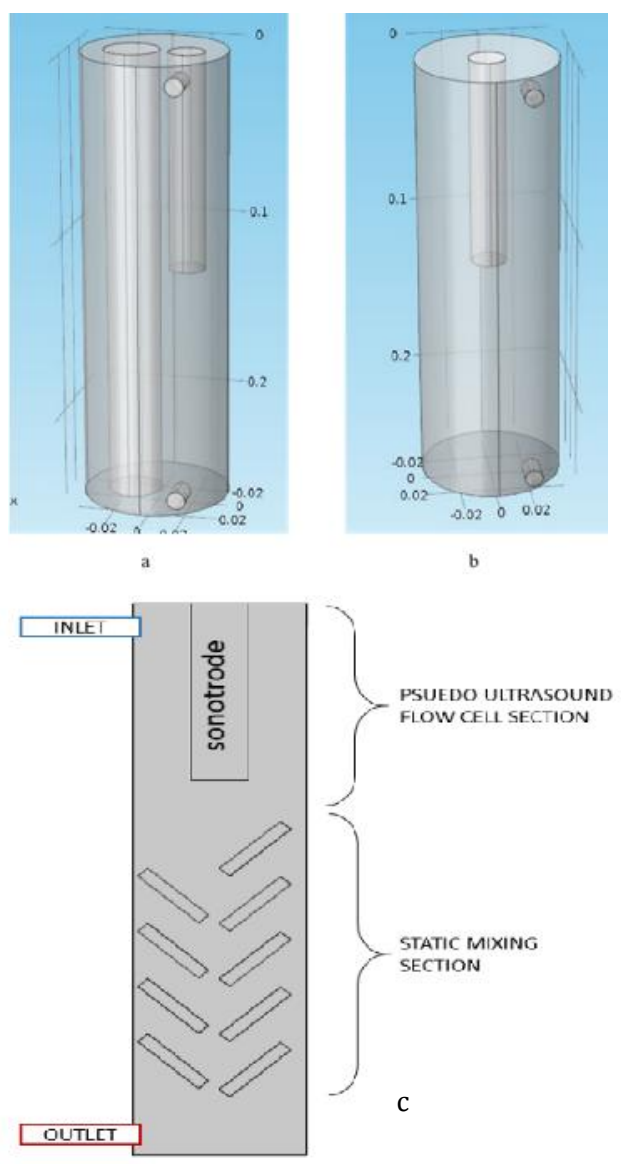

Fig 2. Two different geometries considered for reactor design a) geometry-1, b) geometry-2, c) geometry-3

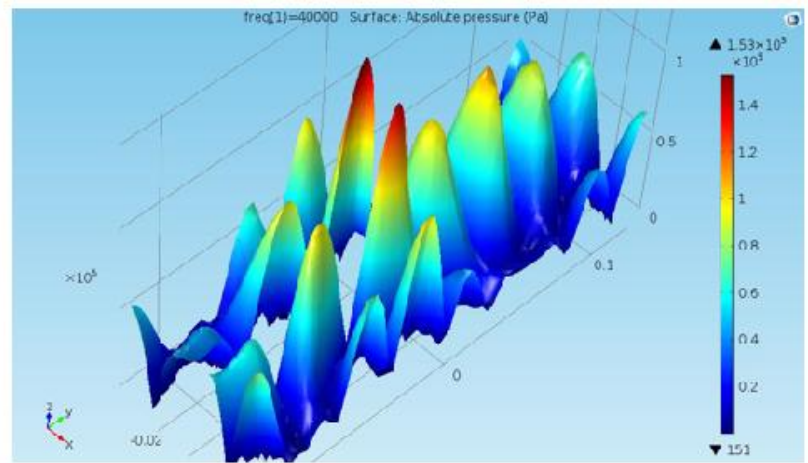

Fig 3. Absolute acoustic pressure profile for reactor geometry 1 and 2 



Fig 4. Pressure profile from General Helmholtz equation a) normal wave number (without cavitation) b) with cavitation

With regard to sonication the first quantity to be evaluated was $\mathrm{T}_{\text {bubble. The highest }} \mathrm{T}_{\text {bubble was }}$ in range of $5555 \mathrm{~K}$ for a liquid temperature of $340 \mathrm{~K}$. T bubble was studied for 3 different liquid temperatures and 3 molar ratios. An interesting observation was made that as molar ratio increased the Tbubble decreased, which could be due to the higher composition of methanol vapors inside the bubble which increase the vapor pressure. At higher molar ratios the localized sonication reaction rate which is the result of implosion of cavitation bubble is decreasing due to decrease in Tbubble. Which implies that for sonication the stoichiometric ratio of the reaction is the best condition for getting better reaction rate. However physically in a global scenario for the fluid, larger molar ratio helps in better mixing of oil and methanol. The reaction kinetics were evaluated for 3 different temperatures, 3 different molar ratios at a constant frequency of $40000 \mathrm{~Hz}$. In each case the $\mathrm{k}_{\text {son }}$ was several orders higher than the $\mathrm{k}_{\text {flow }}$, therefore the reaction rate for sonication was also multiple orders higher. This reaction rate is what occurs at micro level due to implosion of bubbles. This high difference strongly indicates the superiority of sonication over the mechanical agitation process. Fig. 8 (a) the $\mathrm{T}_{\text {bubble }}$ plot for 3 different liquid temperatures and Fig. 8 (b) shows the ln kson vs

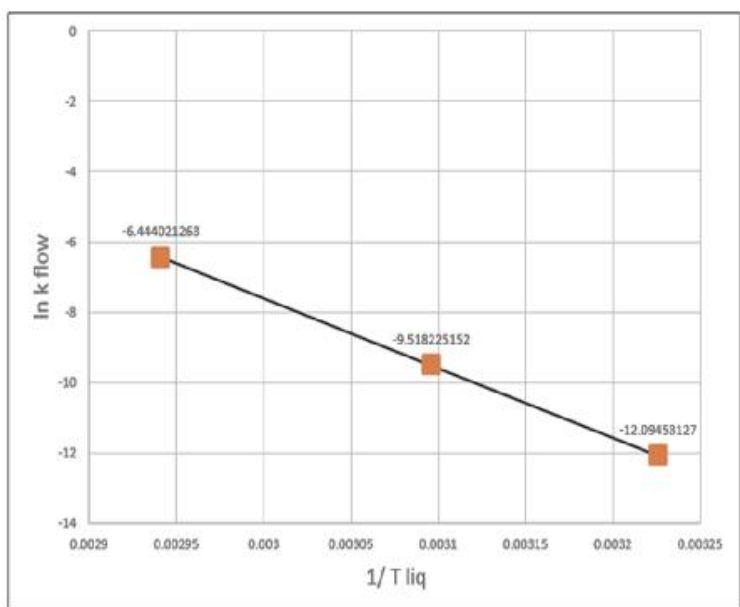

Fig 5. In kflow vs 1/T plot $1 /$ Tbuble plot. $^{2}$

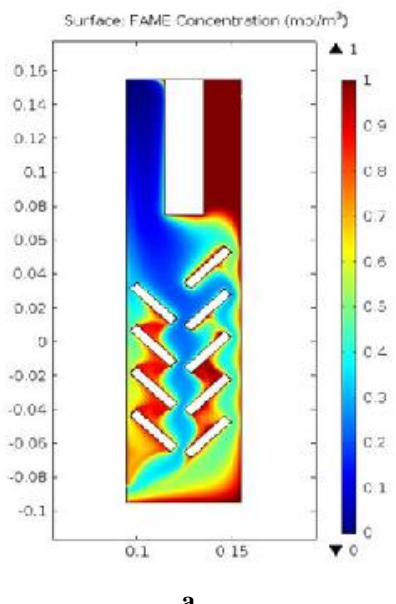

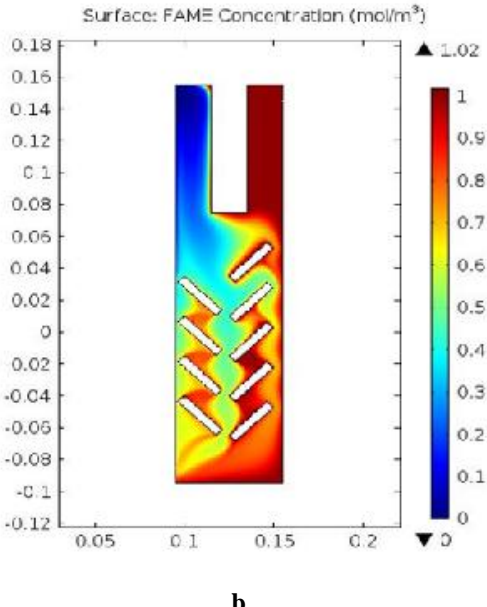

b



c

Fig 6. FAME concentration profile at $340 \mathrm{~K}$ temperature for molar ratio a) 3:1, b) 6:1, c) 9:1 

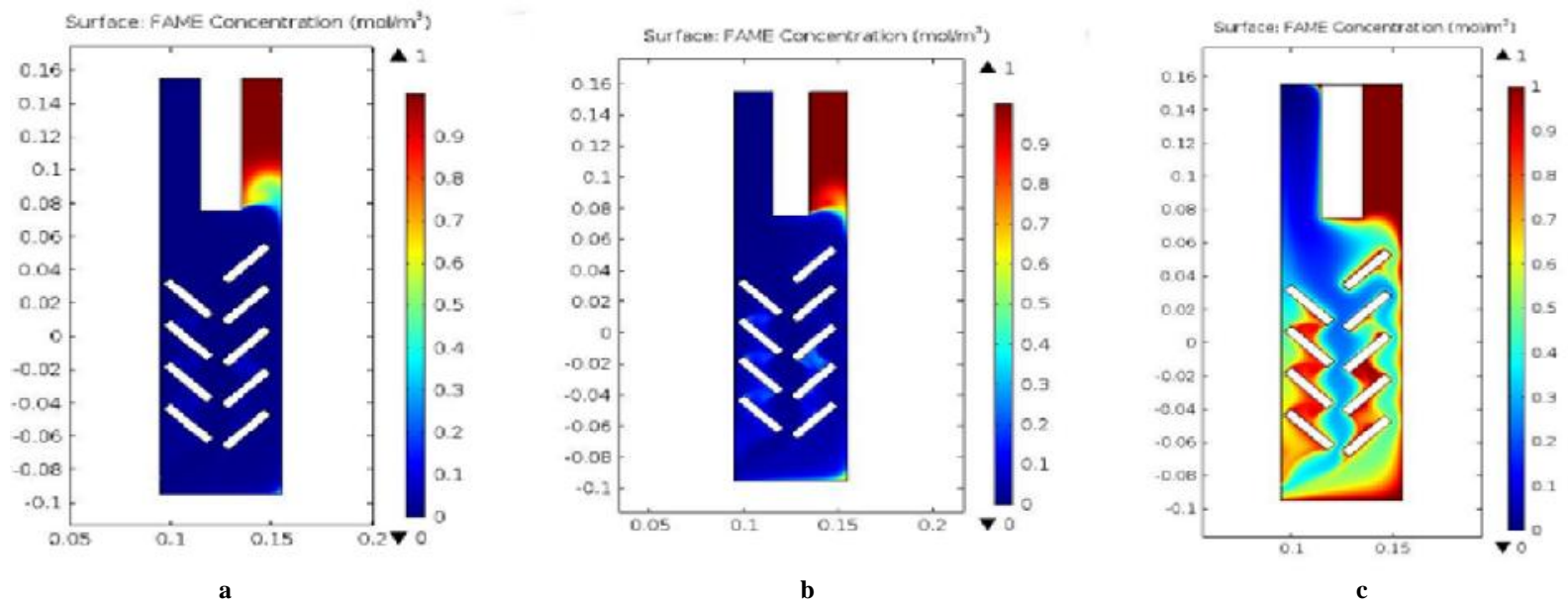

Fig 7. FAME concentration profile at 3:1 molar ratio for temperatures a) $310 \mathrm{~K}$, b) $323 \mathrm{~K}$, c) $340 \mathrm{~K}$

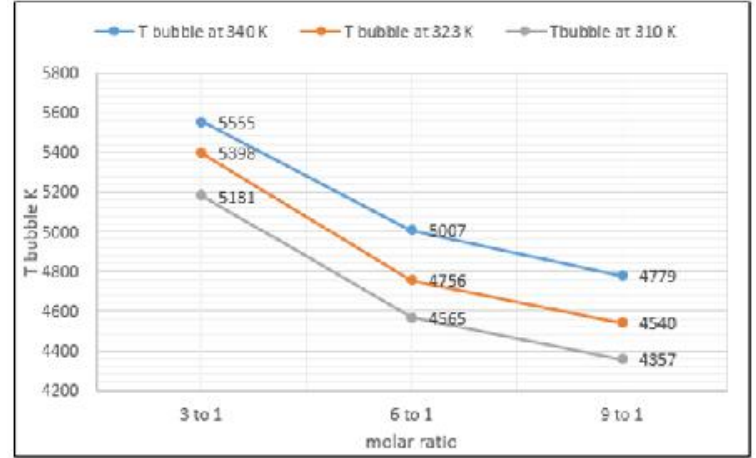

a

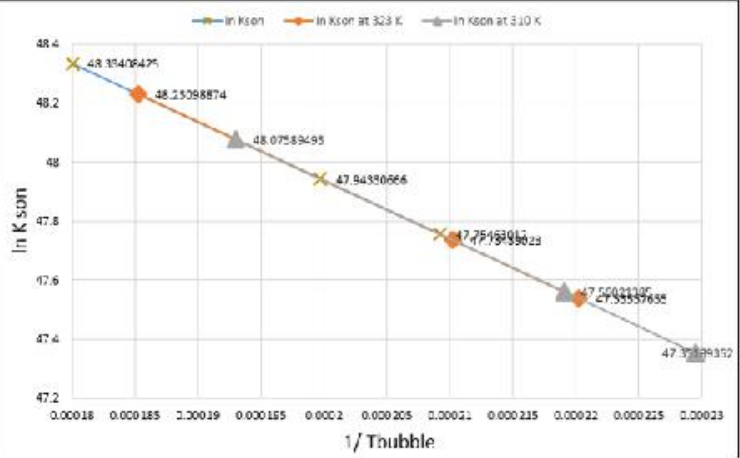

b

Fig 8. a) $T_{\text {bubble }} v s$ molar ratio plot, b) $\ln k_{\text {son }} v s \mathbf{1} / T_{\text {bubble }}$ plot

\section{Conclusion}

The main goal of this work was to optimize the design of a sonochemical reactor used for making biodiesel by the transesterification reaction. Numerical simulation of sonicated, continuous transesterification was done, in which the acoustic analysis was carried out by solving the Helmholtz equation. Cavitation bubble related attenuation was accounted using the complex wave number. Reacting flow was analyzed by solving the Navier Stokes and species transport equations. The design of the reactor had unique sectioning which rendered the part close to the sonicator as a pseudo flow cell, and the bottom half had an incorporated static mixer. Reactive flow analysis showed that having a static mixer section is beneficial based on the observation that the biodiesel concentration was higher than normal between the static mixer blades. Temperature of the bubble area was calculated and used for logical coupling of the reaction rate, i.e. sonication and flow. It was found that the temperature decreases with increase in molar ratio of methanol to oil. This implies that sonication is best when is done at stoichiometric conditions. However, higher molar ratio is good for well mixing of the reactants. The rate constant for sonication was calculated and determined to be multiple orders higher than the rate constant from conventional reaction flow, demonstrating that sonication is much better in terms of other methods. The

reactor gave good yields of FAME in regions away from the sonotrode due to the incorporation of a static mixer. The pseudo flow cell design makes sure that good yields are achieved in the top section of the reactor, which is further enhanced as they trickle down the static mixer. However there is much more scope of optimization which can be done considering different geometry shapes, different static mixer profiles and different ultrasound sources.

\section{Nomenclature}

\section{Property}

Acoustic frequency, $\mathrm{f}$

Activation energy, E

Adiabatic coefficient, $\gamma$

Ambient liquid pressure, $\mathrm{P}_{\text {liq }}$

Blake threshold Pblake

Bulk liquid density, $\rho$ [14]

Dynamic viscosity, [15]

Power applied at the transducers $P_{d}$

$\begin{array}{ll}\text { Unit } & \text { Value } \\ \mathrm{Hz} & 40000 \\ \mathrm{~J} / \mathrm{Mol} & 164958.3 \\ & 8 \\ - & 1.324 \\ \mathrm{~Pa} & 1.00 \mathrm{E}+05 \\ \mathrm{~Pa} & 1.00 \mathrm{E}+05 \\ \mathrm{Kg} / & 883 \\ \mathrm{~m}^{3} & \\ \mathrm{~Pa} . \mathrm{s} & 1.62 \mathrm{E}-02 \\ \mathrm{~W} & 100\end{array}$




\begin{tabular}{|c|c|c|}
\hline Pre-exponential factor A & $\begin{array}{l}\mathrm{m}^{3} / \mathrm{m} \\
\mathrm{ol} / \mathrm{s}\end{array}$ & $3.49 \mathrm{E}+22$ \\
\hline Bubble diameter, $\mathrm{R}=3 / \mathrm{f}[2]$ & M & 0.000075 \\
\hline Speed of sound in liquid, C, [14] & $\mathrm{m} / \mathrm{s}$ & 1920 \\
\hline Surface tension of the liquid, $\sigma[16]$ & $\mathrm{N} / \mathrm{m}$ & 0.003388 \\
\hline Thermal diffusivity of the gas, D [17] & $\mathrm{m}^{2} / \mathrm{s}$ & $9.68 \mathrm{E}-08$ \\
\hline Vapor pressure of vegetable oil [18] & $\mathrm{Pa}$ & 543 \\
\hline Vapor pressure of methanol[19] & $\mathrm{Pa}$ & 13020 \\
\hline Angular frequency $\omega=2 \pi f$ & $\mathrm{rad} / \mathrm{s}$ & 251200 \\
\hline Universal gas constant ${ }_{R u}^{: \pi}$ & $\begin{array}{l}\mathrm{J} / \mathrm{mo} \\
1 . \mathrm{K}\end{array}$ & 8.314 \\
\hline Damping factor, $\mathrm{b}$ & - & - \\
\hline Cavitation bubble volume, $\beta$ & - & - \\
\hline Undisturbed pressure & $\mathrm{Pa}$ & - \\
\hline Resonant frequency, $\omega_{\circ}$ & $\mathrm{rad} / \mathrm{s}$ & - \\
\hline
\end{tabular}

\section{References}

[1] V. G. Gude, G. E. Grant. "Biodiesel from waste cooking oils via direct sonication", Applied Energy, 2013,109, 135-144.

[2] J. Jordens, A. Honings, J. Degrève, L. Braeken, and T. V. Gerven. "Investigation of design parameters in ultrasound reactors with confined channels", Ultrasonics Sonochemistry, 2013, 20, 1345-1352.

[3] J. V. Gerpen. "Biodiesel processing and production," Fuel Processing Technology, 2005, vol. 86, pp. 10971107 .

[4] C. Stavarache, M. Vinatoru, Y. Maeda. "Ultrasonic versus silent methylation of vegetable oils," Ultrasonics Sonochemistry, 2005, vol. 13, pp. 401407, .

[5] S. Manickam, V. N. D. Arigela, P. R. Gogate. "Intensification of synthesis of biodiesel from palm oil using multiple frequency ultrasonic flow cell," Fuel Processing Technology, 2014, vol. 128, pp. 388-393.

[6] R. Jamshidi, B. Pohl, U.A. Peuker, G. Brenner, Numerical investigation of sono-chemical reactors considering the effect of inhomogeneous bubble clouds on ultrasonic wave propagation, Chem. Eng. J. 2012, 189-190, 364-375.

[7] V.S. Sutkar, P.R. Gogate, L. Csoka, Theoretical prediction of cavitational activity distribution in sonochemical reactors, 2010, 158, 290-295.

[8] P.R. Gogate, V.S. Sutkar, A.B. Pandit, Sonochemical reactors: important design and scale up considerations with a special emphasis on heterogeneous systems, Chem. Eng. J. 2011, 166 1066-1082.

[9] I. Janajreh, M. Al Musharrekh and M. Hussain. Numerical simulation of a transesterification and sensitivity study, International Journal of Modern Engineering, 2015 15(2), 13-22

[10] I. Janajreh, M. N. Hussain and T. AlSamad. Assisted Sonication vs Conventional Transesterification numerical simulation and sensitivity study. International conference on advanced Materials (ICAM)，Jordan， 2015，92， DOI:10.1088/1757899X/92/1/012016.

[11] R. E. Caflisch, M. J. Miksis, G. C. Papanicolaou, and L. Ting. Effective equations for wave propagation in bubbly liquids. Journal of Fluid Mechanics, , 1985, 153:259-273.

[12] K.W. Commander, A. Prosperetti, Linear pressure waves in bubbly liquids: comparison between theory and experiments, J. Acoust. Soc. Am. 1988, 85, 732746.

[13] H. Noureddini, and D. Zhu. Kinetics of Transesterification of Soybean Oil. Journal of the American Oil Chemists Society, 1997, 74(11), 14571463.

[14] B. D. Nikoli, B. K., S. D. Markovi, and M. S. Mitrovi, Determining the speed of sound, density, and bulk modulus of rapeseed oil, biodiesel, and diesel fuel, Thermal Science, 2012. 16(2), 505-514

[15] O.O. Fasina and Z. Colley, Viscosity and specific heat of vegetable oils as a function of temperature: $35^{\circ} \mathrm{c}$ to $180^{\circ} \mathrm{c}$, International Journal of Food Properties, 2008, $11,738-746$

[16] E. A. Melo-Espinosa, Y. Sánchez-Borroto, M. Errastia, R. Piloto-Rodríguez, R. Sierens, J. RogerRibac and A C. Hansend, Surface tension prediction of vegetable oils using artificial neural networks and multiple linear regression. Energy Procedia 2014. 57, 886-895.

[17] J. A. B. Lopez, T. M. Alvarado, G. G Coyt, A. M. Diosdado, and J. D. Reyes. Thermal characterization of vegetable oils by means of photo-acoustic techniques. Solar energy, 2013. 59(1), 168-172.

[18] P. M. Ndiaye, F. W. Tavares, I. Dalmolin, C. Dariva, D. Oliveira, and J. V. Oliveira, Vapor Pressure Data of Soybean Oil, Castor Oil, and Their Fatty Acid Ethyl Ester Derivatives. J. Chem. Eng. Data 2005, 50, 330333.

[19] H. F. Gibbard and J. L. Creek, Vapor pressure of methanol from 288.15 to 337.65.deg.K, J. Chem. Eng. Data, 1974, 19 (4), pp 308-310. 